\title{
Atualização no tratamento da hérnia discal cervical: Manejo conservador e indicações de diferentes técnicas cirúrgicas
}

\section{Update on Cervical Hernia Treatment: Conservative Management and Indications of Different Surgical Techniques}

\author{
Luis Eduardo Carelli Teixeira da Silva ${ }^{1,2}$ Luiz Eduardo Pereira Costa Assis de Almeida ${ }^{2}$
}

${ }^{1}$ Instituto Nacional de Traumatologia e Ortopedia (INTO), Rio de Janeiro, RJ, Brasil

2 Instituto da Coluna Vertebral do Rio de Janeiro (INCOL), Rio de Janeiro, RJ, Brasil

Rev Bras Ortop 2021;56(1):18-23.

\author{
Endereço para correspondência Luis Eduardo Carelli Teixeira da Silva, \\ MD, Instituto Nacional de Traumatologia e Ortopedia (INTO), Rua \\ Desembargador João Claudino de Oliveira e Cruz, 50, Apt 1804, Barra \\ da Tijuca, Rio de Janeiro, RJ, 22793-071, Brasil \\ (e-mail: luiscarelli@uol.com.br).
}

\section{Resumo}

\section{Palavras-chave}

- vértebras cervicais

- deslocamento do disco intervertebral/ diagnóstico

- deslocamento do disco intervertebral/terapia

- deslocamento do disco intervertebral/cirurgia
A dor cervical e radicular devido à hérnia de disco é uma causa comum de uma visita a um cirurgião ortopédico. É importante saber diagnosticar, tratar e inicialmente. Quais são as melhores opções para tratar a hérnia de disco hoje em dia? Este artigo faz uma revisão da literatura e atualização sobre o tratamento clínico e cirúrgico da herniação do disco cervical.

\section{Abstract \\ Keywords \\ - cervical vertebrae \\ - intervertebral disc displacement/ diagnosis \\ - intervertebral disc displacement/ therapy \\ - intervertebral disc displacement/surgery}

Cervical and root pain due to herniated disc is a common cause of a visit to an orthopedic surgeon. It is important to know how to diagnose, treat and initially. What are the best options to treat a herniated disc nowadays? The present article reviews the literature and updates on the clinical and surgical treatment of cervical disc herniation. recebido

01 de Junho de 2020

aceito

16 de Setembro de 2020
DOI https://doi.org/

$10.1055 / \mathrm{s}-0041-1724007$. ISSN $0102-3616$

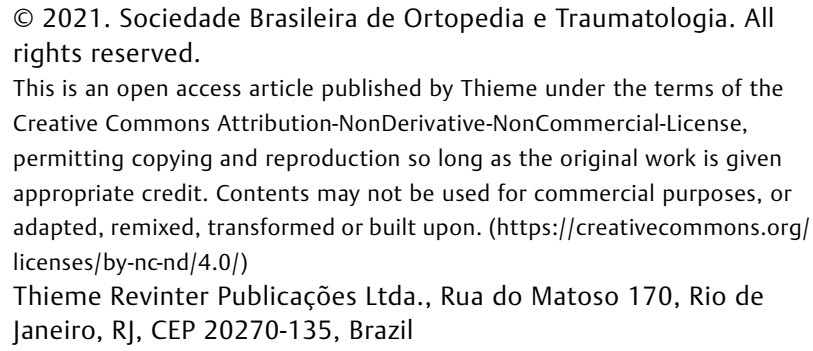

(c) 2021. Sociedade Brasileira de Ortopedia e Traumatologia. All rights reserved.

This is an open access article published by Thieme under the terms of the Creative Commons Attribution-NonDerivative-NonCommercial-License, permitting copying and reproduction so long as the original work is given appropriate credit. Contents may not be used for commercial purposes, or adapted, remixed, transformed or built upon. (https://creativecommons.org/ licenses/by-nc-nd/4.0/)

Thieme Revinter Publicações Ltda., Rua do Matoso 170, Rio de Janeiro, RJ, CEP 20270-135, Brazil 


\section{Introdução}

A doença discal cervical pode ter diferentes formas de apresentação, tais como a cervicalgia, radiculopatia e mielopatia, sendo importante essa diferenciação pelo médico ortopedista para guiar o tratamento.

A cervicalgia é o sintoma mais prevalente da síndrome discal cervical, apresentando uma prevalência durante a vida de 48,5 a $66,7 \%$, dependendo do estudo analisado. ${ }^{1,2}$

A radiculopatia cervical pode ser de etiologia compressiva ou inflamatória, pelas citocinas liberadas devido à hérnia discal e ruptura do ânulo fibroso. ${ }^{3}$ Pode apresentar-se como braquialgia, alteração sensitiva, alteração motora, dor escapular e cefaleia. ${ }^{4}$

Já a mielopatia causada pela hérnia discal com compressão anterior da medula pode apresentar alterações da marcha e esfincterianas além de sinais de acometimento de primeiro neurônio motor.

O processo fisiopatológico que engloba a doença discal cervical tem início na terceira década de vida, com a diminuição da vascularização discal e aumento da esclerose das placas terminais, facilitando o desgaste discal e diminuindo a formação de proteoglicanos. Uma vez iniciado esse processo, ocorre alteração da absorção de carga pelo disco, promovendo fissuras do ânulo e, por fim, a hérnia discal ou o colapso. ${ }^{5,6}$

É importante ressaltar que o exame físico nos fornece informações valiosas na investigação etiológica e na localização da doença na coluna cervical.

Devemos sempre caracterizar a dor cervical, se ela tem contratura muscular associada, se a dor piora durante a extensão ou flexão cervical, e o tempo de curso da dor.

A dor radicular geralmente segue um padrão de distribuição no exame (-Tabela $\mathbf{1}$ ), que deverá ser investigado passo a passo e pode apresentar piora ao teste de Spurling e melhora na abdução do ombro.

Procurar achados de mielopatia é imprescindível já que, não raro, pode demorar a apresentar sintomas, e o desfecho pode ser catastrófico. Devemos sempre avaliar os reflexos, a marcha, o trofismo muscular e a presença do sinal de Hoffman.

Os exames complementares disponíveis para confirmação diagnóstica e entendimento da doença cervical em questão são: radiografia nas incidências anteroposterior, perfil, e perfil em flexão e em extensão; tomografia computadorizada; e ressonância magnética.

A discografia provocativa, utilizada no passado, está sendo cada vez menos indicada devido a sua acurácia limitada, com altas taxas de falso positivo, podendo chegar até $50 \%$, e com a possibilidade de degeneração precoce nos níveis controles. ${ }^{7}$
Nos últimos anos, com o melhor entendimento do alinhamento sagital cervical e global na promoção das doenças cervicais, no planejamento cirúrgico e a sua correlação com o resultado clínico, as radiografias da coluna cervical e panorâmica se tornaram ferramentas importantes, necessitando realizar medidas radiológicas além da lordose cervical, tais como: o eixo sagital vertical (ESV) da coluna cervical, inclinação de T1 e versão cervical (neck tilt), parâmetros estes análogos aos descritos para o alinhamento espino-pélvico ${ }^{8,9}$ ( - Figura 1).

\section{Tratamento}

Existem várias opções de tratamento para a hérnia discal cervical, entre elas o tratamento medicamentoso; o tratamento não intervencionista, que engloba a fisioterapia, acupuntura, imobilização, tração; o tratamento intervencionista da dor, que inclui os bloqueios neurais e facetários; e o tratamento cirúrgico, que é indicado nos casos de radiculopatia intratável ou mielopatia $^{7}$ (Quadro 1).

Fazendo uma analogia com o tratamento conservador medicamentoso para a lombociatalgia, as melhores opções são os analgésicos comuns, como o parecetamol e a dipirona associados ou não aos opioides, e o uso orientado de antiinflamatórios não esteroides (AINEs). Deve-se evitar o uso de corticoide oral, que não mostrou ter benefícios no controle da dor radicular. ${ }^{10}$

Nos dias atuais, a tração esquelética controlada e os exercícios de fortalecimento continuam sendo um dos melhores métodos para o alívio da dor a curto e médio prazo, sendo mais eficientes que $o$ alongamento. A tração esquelética pode ser realizada em ventroflexão ou em leve extensão cervical, inicialmente com 4 a $7 \mathrm{~kg}$ e com possível incremento de carga. Não foram vistas complicações graves com o uso da tração. ${ }^{11-13}$

\section{Tratamento intervencionista}

Nos casos refratários ao tratamento conservador, podem ser realizados bloqueios terapêuticos para controle da dor radicular e axial. Basicamente, são utilizados três tipos de bloqueio: bloqueio seletivo foraminal, bloqueio epidural translaminar e bloqueios facetários.

O bloqueio seletivo foraminal guiado por fluoroscopia apresenta boa efetividade no tratamento da dor radicular, com taxas de sucesso de alívio da dor em torno de $81 \%$ da braquialgia e de $66 \%$ da cervicalgia, podendo, assim, evitar cirurgias. Ocorrências de complicações graves são pouco relatadas; consequentemente, esses procedimentos podem ser considerados seguros.

Tabela 1 Distribuição da disfunção radicular cérvico-braquial

\begin{tabular}{|l|l|l|l|l|}
\hline Raiz & Localização da dor & Disfunção motora & Disfunção sensitiva & Reflexo \\
\hline C5 & Ombro e braço & $\begin{array}{l}\text { Deltoide, supraespinhoso, } \\
\text { infraespinhoso e bíceps. }\end{array}$ & Proximal e lateral do ombro & Biciptal \\
\hline C6 & Porção radial do antebraço & Bíceps e extensores do punho & Porção radial do antebraço & Estilorradial \\
\hline C7 & Porção dorsal do antebraço & Tríceps e flexores do punho & Dedo indicador e médio & Triciptal \\
\hline C8 & Porção ulnar do antebraço & Intrínsecos da mão & Anelar e borda ulnar da mão & - \\
\hline
\end{tabular}



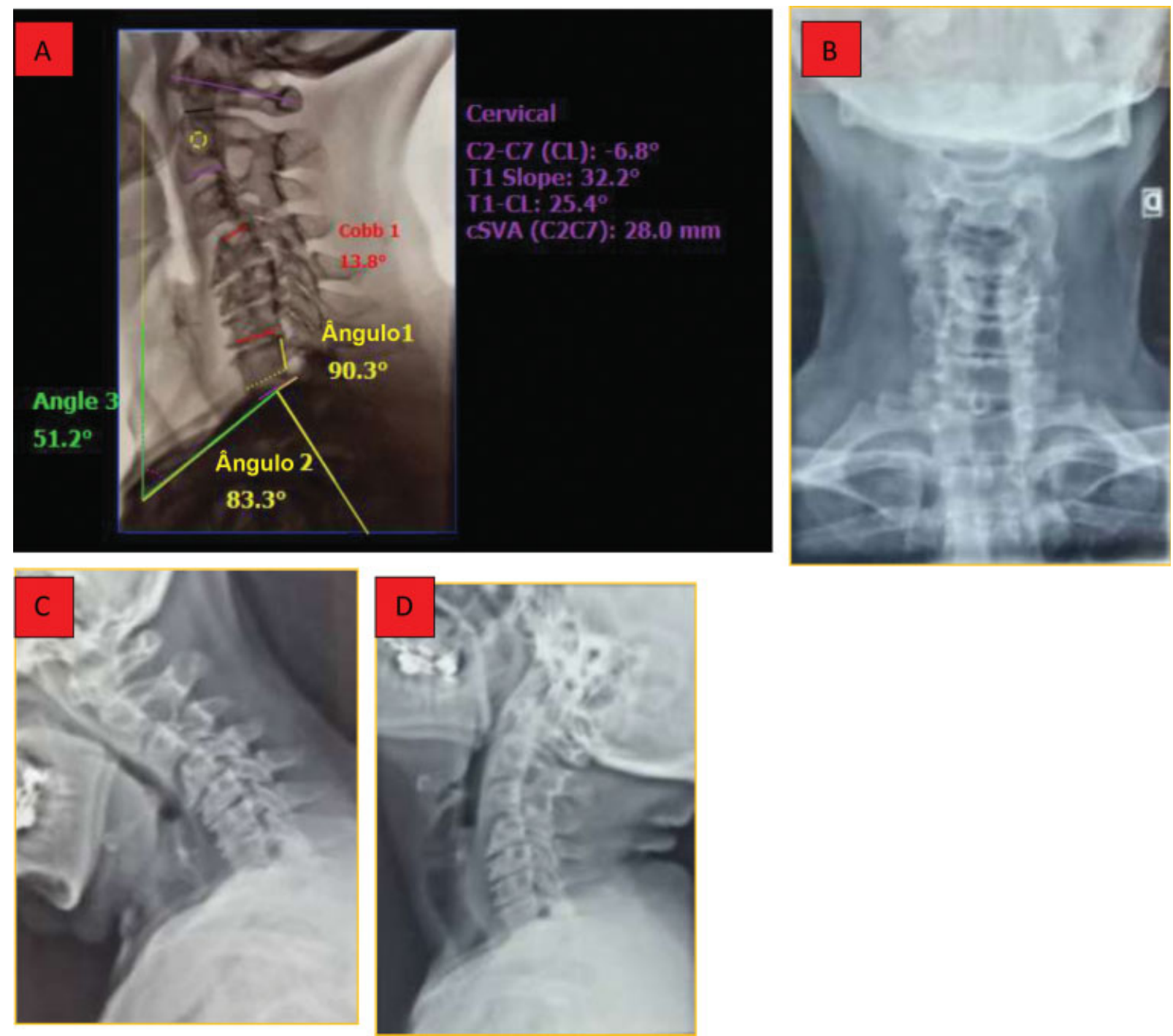

Fig. 1 Parâmetros radiográficos de alinhamento sagital cervical. (A) Radiografia em perfil com medições do alinhamento sagital da coluna cervical; (B) Radiografia em incidência anteroposterior; (C) Radiografia em perfil com flexão cervical; (D) Radiografia em perfil com extensão cervical; (E) Tomografia computadorizada da coluna cervical; (F-G) Ressonância magnética da coluna cervical.

Quadro 1 Opções de tratamento de hérnia discal cervical

\begin{tabular}{|l|l|l|}
\hline Tratamento conservador & Tratamento intervencionista & Tratamento cirúrgico \\
\hline $\begin{array}{l}\text { - Medicamentoso } \\
\text { - Fisioterapia }\end{array}$ & $\begin{array}{l}\text { - Bloqueio foraminal } \\
\text { - Tração } \\
\text { - Fortalecimento }\end{array}$ & $\begin{array}{l}\text { - Discetomia posterior + foraminotomia } \\
\text { - Discectomia anterior + artrodese } \\
\text { - Artroplastia discal cervical }\end{array}$ \\
\hline
\end{tabular}

Já o bloqueio cervical epidural por via interlaminar, de acordo com a literatura, apresenta taxa de sucesso moderada para alívio da dor radicular e, com alguns relatos de quadriparesia ou lesão medular cervical, é, portanto, proscrito para uso rotineiro. ${ }^{14-18}$

\section{Tratamento cirúrgico}

O tratamento cirúrgico da hérnia discal cervical está indicado nos casos de falha do tratamento conservador ou por sinais e sintomas de compressão mieloradicular, ocasionando dor excruciante, recorrente, ou déficit neurológico importante ou compressivo.

O principal objetivo a ser alcançado, independente da técnica cirúrgica a ser utilizada, é a descompressão das estruturas neurais.

Devemos ressaltar que as evidências atuais não suportam o tratamento cirúrgico da dor axial sem sintomas radiculares, seja por artrodese ou artroplastia discal cervical, por apresentarem resultados clínicos insatisfatórios na região subaxial e, portanto, devemos reservar o tratamento cirúrgico na presença de síndromes compressivas medulares ou radiculares. 
Entre as opções de tratamento cirúrgico disponíveis, o cirurgião poderá escolher dentre as seguintes técnicas: Discectomia posterior associada a foraminotomia, aberta ou minimamente invasiva; abordagens cervicais anteriores para descompressão associada ou não à artrodese cervical; ou utilização de artroplastias do disco cervical.

\section{Discectomia posterior}

A discectomia posterior, abandonada no passado devido às complicações neurológicas e agressão tecidual em cirurgias abertas, está recuperando espaço em casos selecionados de herniações póstero-laterais ou foraminais que cursam com radiculopatia, utilizando métodos minimamente invasivos com afastadores tubulares ou endoscópicos.

A descompressão foraminal posterior, descrita por Scoville em 1944, tem sido aprimorada; no entanto, os preceitos básicos de preservação parcial de facetas articulares para evitar instabilidade, além das contraindicações para o seu uso nas hérnias de disco centrais e calcificação do ligamento longitudinal posterior devem ser respeitadas.

Desta maneira, o uso dos endoscópicos para esta finalidade tem se mostrado eficaz, com redução de 87 a $97 \%$ da dor radicular. $^{19-23}$

Evidências atuais sugerem que talvez os riscos da cirurgia minimamente invasiva não superem os benefícios e que o uso rotineiro destas técnicas requeira estudos mais detalhados e com melhor nível de evidência. Contudo, com o adequado treinamento e seleção criteriosa de pacientes, as novas tecnologias têm se mostrado seguras e promissoras. ${ }^{24}$

\section{Discectomia cervical anterior e artrodese}

Em1958, Cloward descreveu a primeira discectomia e artrodese cervical anterior com enxerto estrutural de crista ilíaca e, respeitadas as devidas evoluções na técnica, esta ainda é amplamente reconhecida como o padrão-ouro para o trata- mento cirúrgico da hérnia de disco cervical (-Figura 2). Atualmente, são utilizados espaçadores (cages) de titânio ou em polieteretercetona ( $\mathrm{PEEK}$, na sigla em inglês) e placas, com o objetivo de assegurar maior estabilidade, restaurar lordose cervical, diminuir taxas de subsidência e aumentar a osteointegração. ${ }^{25,26}$

Estudos recentes randomizados, de revisão sistemática e de meta-análise, comparando utilização de cages isolados (stand-alone) e cirurgias com uso de cages e placas, demonstraram vantagens na utilização adjunta de placas e parafusos, tais como: maior estabilidade biomecânica imediata, maiores taxas de artrodese, melhora da lordose cervical, melhora da cervicalgia e menores taxas de subsidência. No entanto, complicações não estatisticamente significativas podem ocorrer com maior frequência quando se utiliza placas e parafusos, como disfagia e falha de material de síntese.

Apesar da melhora da dor cervical nos trabalhos com a utilização de placa, os resultados funcionais são similares nos dois grupos. $^{27}$

Com o objetivo de diminuir a agressão tecidual, disfagia e a síndrome do nível adjacente, foram desenvolvidos cages bloqueados utilizando âncoras ou parafusos.

$\mathrm{Na}$ literatura atual, trabalhos que demonstram a utilização de cages bloqueados comparados à placa, esses apresentam menor tempo cirúrgico, perda sanguínea e menor incidência de disfagia no pós-operatório tardio, bem como menor ossificação do nível adjacente. Entretanto, a avaliação dos resultados clínicos, funcionais, radiológicos e as taxas de subsidência se mostraram semelhantes em ambas as técnicas. ${ }^{28,29}$

Nos pacientes com desalinhamento do plano sagital cervical e cervicobraquialgia, a manutenção ou melhora do alinhamento cervical, principalmente da lordose cervical, da inclinação de T1 (T1 slope) e da discrepância da lordose cervical com a inclinação de T1 (CL-TS), mostrou ter boa correlação com os resultados clínicos e funcionais, sendo a artrodese o mais indicado nestes casos. ${ }^{30}$
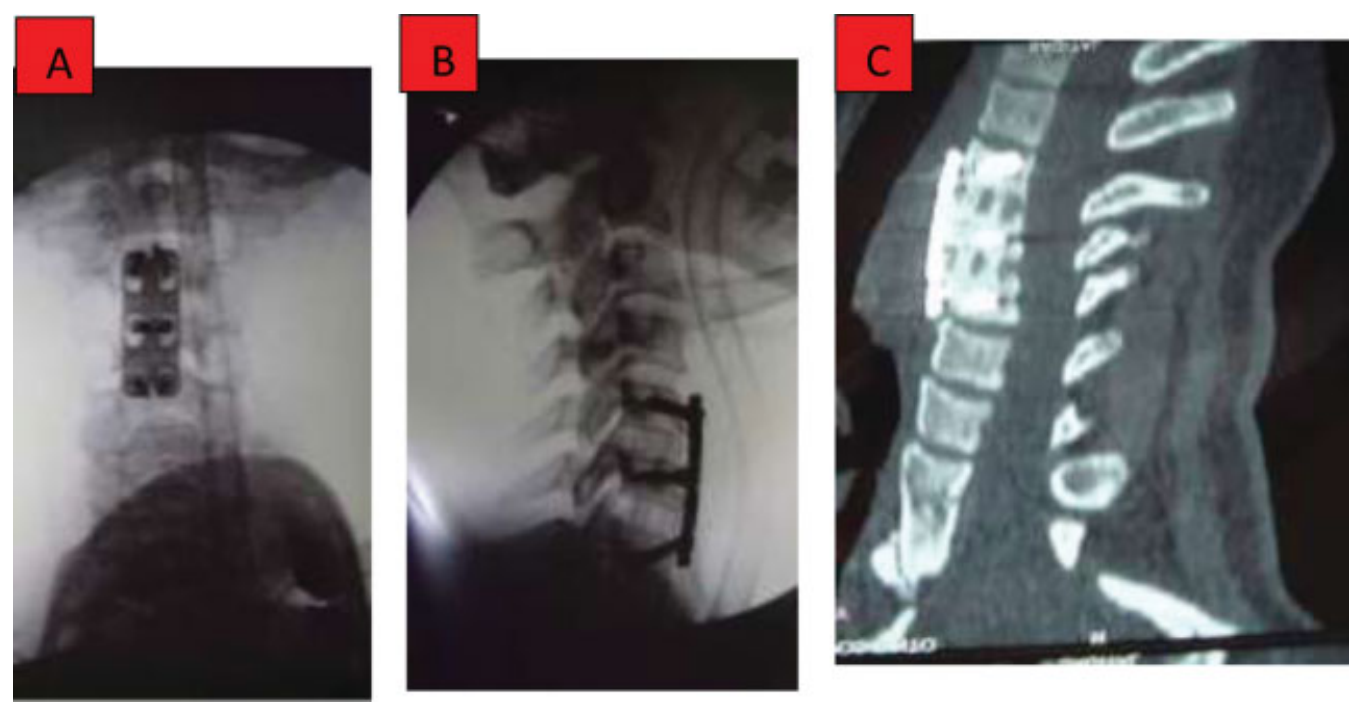

Fig. 2 Artrodese cervical anterior com placa e cage em polieteretercetona. (A) Imagem intraoperatória em incidência anteroposterior; (B) Imagem intraoperatória em perfil; (C) Tomografia computadorizada evidenciando consolidação. 


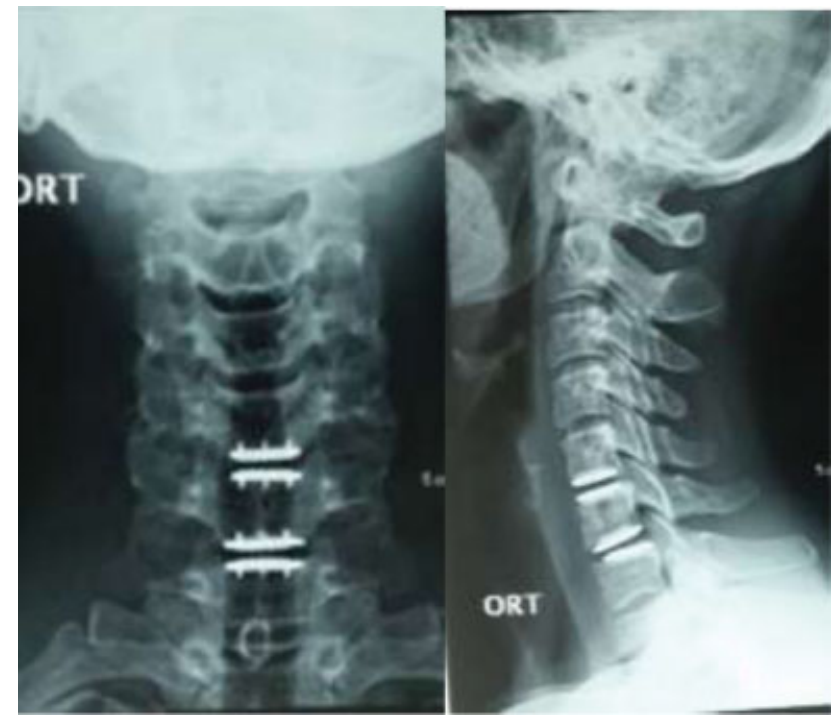

Fig. 3 Artroplastia discal cervical. (A) Radiografia em incidência anteroposterior; (B) Radiografia em perfil.

\section{Artroplastia}

Com o intuito de preservação da mobilidade cervical no nível operado, prevenção de síndrome do nível adjacente e melhora de resultados clínicos, a artroplastia do disco intervertebral cervical vem sendo utilizada nos últimos anos (-Figura 3).

Inúmeros modelos de próteses, sejam elas constritas, não constritas e semiconstritas; confeccionadas de metal- metal ou com núcleos de poliuretano, todas elas mantêm as mesmas indicações da artrodese cervical anterior, apresentando as seguintes contraindicações: Cirurgia em três ou mais níveis, instabilidade cervical, osteopenia, infecção ativa e deformidade cifótica. ${ }^{31}$

Alguns trabalhos demonstram a superioridade da prótese, indicando a redução da incidência de reoperação no nível operado, a diminuição de síndrome do nível adjacente e a manutenção do arco de movimento da coluna cervical como vantagens, apesar do aparecimento de ossificação heterotópica relevante, que ocorre em torno de $23 \%$ dos casos, sem comprometer os melhores resultados clínicos e funcionais quando comparados à artrodese cervical anterior. ${ }^{32}$

Contudo, outros estudos randomizados questionam a efetividade da artroplastia quando comparada à discectomia cervical anterior associada ou não à fusão intersomática, com resultados funcionais semelhantes entre as técnicas, não evidenciando superioridade de uma em relação a outra. ${ }^{33}$

\section{Considerações finais}

Pacientes portadores de hérnia de disco cervical podem apresentar uma miríade de achados radiológicos que têm ou não correlação clínica. Na falha do tratamento conservador, os bloqueios guiados por imagem podem ser empregados para o tratamento de radiculopatia, e, caso haja necessidade de intervenção cirúrgica, deve ser realizado um planejamento correto, baseado na presença de discopatia, mielopatia, alinhamento cervical e presença ou não de instabilidade.

Após uma criteriosa avaliação do quadro clínico, a escolha do procedimento cirúrgico deve ser baseada em: recursos hospitalares disponíveis, experiência do cirurgião, e literatura com melhor nível de evidência; desta maneira, será possível indicar a melhor técnica cirúrgica: discetomia/foraminotomia posterior, artrodese anterior ou artroplastia.

\section{Conflito de interesses}

Os autores declaram não haver nenhum conflito de interesses.

\section{Referências}

1 Côté P, Cassidy JD, Carroll L. The Saskatchewan Health and Back Pain Survey. The prevalence of neck pain and related disability in Saskatchewan adults. Spine 1998;23(15):1689-1698

2 Fejer R, Kyvik KO, Hartvigsen J. The prevalence of neck pain in the world population: a systematic critical review of the literature. Eur Spine J 2006;15(06):834-848

3 Kang JD, Georgescu HI, McIntyre-Larkin L, Stefanovic-Racic M, Evans $\mathrm{CH}$. Herniated cervical intervertebral discs spontaneously produce matrix metalloproteinases, nitric oxide, interleukin-6, and prostaglandin E2. Spine 1995;20(22):2373-2378

4 Hoy DG, Protani M, De R, Buchbinder R. The epidemiology of neck pain. Best Pract Res Clin Rheumatol 2010;24(06):783-792

5 Clark CR, Benzel EC, Eds. The cervical spine. Philadelphia: Lippincott Williams \& Wilkins; 2005

6 Taylor TK, Melrose J, Burkhardt D, et al. Spinal biomechanics and aging are major determinants of the proteoglycan metabolism of intervertebral disc cells. Spine 2000;25(23):3014-3020

7 Manchikanti L, Abdi S, Atluri S, et al. An update of comprehensive evidence-based guidelines for interventional techniques in chronic spinal pain. Part II: guidance and recommendations. Pain Physician 2013;16(2, Suppl)S49-S283

8 Pratali RR, Hennemann SA, Amaral R, et al. Terminologia padronizada da deformidade vertebral do adulto para o português do Brasil. Coluna/Columna 2015;14(04):281-285

9 Ames CP, Blondel B, Scheer JK, et al. Cervical radiographical alignment: comprehensive assessment techniques and potential importance in cervical myelopathy. Spine 2013;38(22, Suppl 1): S149-S160

10 Goldberg H, Firtch W, Tyburski M, et al. Oral steroids for acute radiculopathy due to a herniated lumbar disk: a randomized clinical trial. JAMA 2015;313(19):1915-1923

11 Moustafa IM, Diab AA. Multimodal treatment program comparing 2 different traction approaches for patients with discogenic cervical radiculopathy: a randomized controlled trial. J Chiropr Med 2014;13(03):157-167

12 Yang JD, Tam KW, Huang TW, Huang SW, Liou TH, Chen HC. Intermittent Cervical Traction for Treating Neck Pain: A Metaanalysis of Randomized Controlled Trials. Spine 2017;42(13): 959-965

13 Ylinen J, Takala EP, Nykänen M, et al. Active neck muscle training in the treatment of chronic neck pain in women: a randomized controlled trial. JAMA 2003;289(19):2509-2516

14 Ma DJ, Gilula LA, Riew KD. Complications of fluoroscopically guided extraforaminal cervical nerve blocks. An analysis of 1036 injections. J Bone Joint Surg Am 2005;87(05):1025-1030

15 Abbasi A, Malhotra G, Malanga G, Elovic EP, Kahn S. Complications of interlaminar cervical epidural steroid injections: a review of the literature. Spine 2007;32(19):2144-2151

16 Bose B. Quadriparesis following cervical epidural steroid injections: case report and review of the literature. Spine J 2005;5(05): $558-563$ 
17 Hodges SD, Castleberg RL, Miller T, Ward R, Thornburg C. Cervical epidural steroid injection with intrinsic spinal cord damage. Two case reports. Spine 1998;23(19):2137-2142, discussion 2141-2142

18 Ludwig MA, Burns SP. Spinal cord infarction following cervical transforaminal epidural injection: a case report. Spine 2005;30 (10):E266-E268

19 Scoville WB. Recent developments in the diagnosis and treatment of cervical ruptured intervertebral discs. Proc Am Fed Clin Res 1945;2:23

20 Song Z, Zhang Z, Hao J, et al. Microsurgery or open cervical foraminotomy for cervical radiculopathy? A systematic review. Int Orthop 2016;40(06):1335-1343

21 O’Toole JE, Sheikh H, Eichholz KM, Fessler RG, Perez-Cruet MJ. Endoscopic posterior cervical foraminotomy and discectomy. Neurosurg Clin N Am 2006;17(04):411-422

22 Gala VC, O’Toole JE, Voyadzis JM, Fessler RG. Posterior minimally invasive approaches for the cervical spine. Orthop Clin North Am 2007;38(03):339-349, abstract v

23 Zdeblick TA, Zou D, Warden KE, McCabe R, Kunz D, Vanderby R. Cervical stability after foraminotomy. A biomechanical in vitro analysis. J Bone Joint Surg Am 1992;74(01):22-27

24 Evaniew N, Khan M, Drew B, Kwok D, Bhandari M, Ghert M. Minimally invasive versus open surgery for cervical and lumbar discectomy: a systematic review and meta-analysis. CMAJ Open 2014;2(04):E295-E305

25 Cloward RB. The anterior approach for removal of ruptured cervical disks. J Neurosurg 1958;15(06):602-617

26 Song KJ, Taghavi CE, Lee KB, Song JH, Eun JP. The efficacy of plate construct augmentation versus cage alone in anterior cervical fusion. Spine 2009;34(26):2886-2892
27 Oliver JD, Goncalves S, Kerezoudis P, et al. Comparison of Outcomes for Anterior Cervical Discectomy and Fusion With and Without Anterior Plate Fixation: A Systematic Review and MetaAnalysis. Spine 2018;43(07):E413-E422

28 Nambiar M, Phan K, Cunningham JE, Yang Y, Turner PL, Mobbs R. Locking stand-alone cages versus anterior plate constructs in single-level fusion for degenerative cervical disease: a systematic review and meta-analysis. Eur Spine J 2017;26(09): 2258-2266

29 Nemoto O, Kitada A, Naitou S, Tachibana A, Ito Y, Fujikawa A. Stand-alone anchored cage versus cage with plating for singlelevel anterior cervical discectomy and fusion: a prospective, randomized, controlled study with a 2-year follow-up. Eur J Orthop Surg Traumatol 2015;25(Suppl 1):S127-S134

30 Iyer S, Nemani VM, Nguyen J, et al. Impact of Cervical Sagittal Alignment Parameters on Neck Disability. Spine 2016;41(05): 371-377

31 Koreckij TD, Gandhi SD, Park DK. Cervical Disk Arthroplasty. J Am Acad Orthop Surg 2019;27(03):e96-e104

32 Hisey MS, Bae HW, Davis RJ, et al. Prospective, Randomized Comparison of Cervical Total Disk Replacement Versus Anterior Cervical Fusion: Results at 48 Months Follow-up. J Spinal Disord Tech 2015;28(04):E237-E243

33 Vleggeert-Lankamp CLA, Janssen TMH, van Zwet E, et al. The NECK trial: Effectiveness of anterior cervical discectomy with or without interbody fusion and arthroplasty in the treatment of cervical disc herniation; a double-blinded randomized controlled trial. Spine J 2019;19(06):965-975 